steuerten Handeln von Staaten theoretische Konsequenzen zu ziehen, liefern die Referate, die überwiegend versuchen, aus den neueren Entwicklungen gerade in Europa seit 1989 auch theoretische Schlußfolgerungen zu ziehen, einen wertvollen Beitrag zur Durchdringung eines - gemessen an seinen praktischen Konsequenzen im Ernstfall - immer noch viel zu wenig erfaßten Bereichs des Völkerrechts.

Christiane Simmler

\title{
Oliver Dörr
}

\section{Die Inkorporation als Tatbestandsmerkmal der Staatensukzession}

Schriften zum Völkerrecht, Band 120

Duncker \& Humblot Verlag, Berlin, 1995, 434 S., DM 124,--

Die Berliner Dissertation widmet sich mit dem Problemfeld der Staatensukzession einer Materie des Völkerrechts, die mit der Bezeichnung als "unklar und schwierig" (S. 21) noch vorsichtig charakterisiert ist. Gerade die umstürzenden Veränderungen in der Staatenlandschaft Südost- und Osteuropas haben vor Augen geführt, wie wenig dieses für den Fortbestand der Staaten als Völkerrechtssubjekte so wichtige Thema von der Wissenschaft theoretisch durchdrungen ist und (vielleicht gerade deswegen) in der Praxis stringente Anwendung findet. Die nunmehr wieder (nach längerer Ruhephase) hohe Aktualität der Probleme der Staatensukzession macht das Unternehmen, einen Teilbereich der Staatensukzession im Detail zu beleuchten und dogmatisch zu erfassen, besonders verdienstvoll. Dabei weicht die erste Verwunderung über den eher eng gefaßten Untersuchungsgegenstand, den Tatbestand der Inkorporation unter Ausklammerung der Rechtsfolgen, schnell der Erkenntnis, daß angesichts des lobenswerten Ansatzes, die Untersuchung des Tatbestands der Inkorporation entgegen früheren Abhandlungen auf eine sehr umfangreiche Prüfung der Staatenpraxis zu stützen, allein diese Beschränkung ein kaum beherrschbares Ausufern der Arbeit verhindern konnte.

Bereits in der Einleitung erfolgt nach einer kurzen Einführung in die Problemstellung eine Erläuterung des Begriffs der Staatensukzession für den Rahmen dieser Arbeit: Es wird auf den "eigentlichen Vorgang der Gebietsveränderung" (S. 27) abgestellt, als "Staatensukzession" (respektive "Staatennachfolge") ist der "Übergang der territorialen Souveränität in bezug auf ein bestimmtes Gebiet von einem Staat auf einen anderen" (S. 30) zu verstehen. Angesichts des auch in der vorliegenden Studie beklagten erheblichen Terminologiewirrwarrs in Lehre und Praxis in allen die Veränderung bestehender Staaten betreffenden Bereichen des Völkerrechts ist die frühe terminologische Klarstellung erfreulich.

Die weitere Darstellung zerfällt in zwei Teile. Zunächst wird der "Tatbestand der Inkorporation" (ab S. 31) beleuchtet, dann werden "Historische Beispiele" (ab S. 191) auf ihre Subsumierbarkeit unter den ausgearbeiteten Inkorporationstatbestand untersucht. Der erste 
Teil beginnt mit einer Präsentation der "Grundkonstellation" der Inkorporation als der "vollständigen Eingliederung eines souveränen Staates in einen anderen" (S. 31), die als ein Tatbestand der Staatensukzession entsprechend der oben wiedergegebenen Definition verstanden wird. Dabei arbeitet der Autor eine eigene Definition der Inkorporation heraus: den "Untergang eines souveränen Staates als geborenes Völkerrechtssubjekt durch seine vollständige Aufnahme in einen anderen Staatsverband, der schon vorher bestanden hat" (S. 39). Kurz wird auf den Einfluß des völkerrechtlichen Effektivitätsprinzips eingegangen und betont, daß eine effektive Inkorporation die Konstruktion von "wiedererstehenden Staaten" verbietet, wie sie nach dem Zerfall der UdSSR durch die Wiedererrichtung der baltischen Staaten von vielen Seiten propagiert wird. Der Definition folgt eine Abgrenzung zur Annexion (ab S. 91), die vom Merkmal der Freiwilligkeit (d.h. insbesondere Gewaltfreiheit) der Aufnahme in den anderen Staatsverband ausgeht. Im Rahmen einer umfassenden Auseinandersetzung mit dem Rechtsinstitut der Annexion und der historischen Entwicklung des Gewaltverbots im modernen Völkerrecht kommt der Verfasser zu dem Ergebnis, daß seit Ende der sechziger Jahre von der Staatengemeinschaft gewaltsamer Gebietserwerb nicht mehr hingenommen wird, so daß die Annexion als Sukzessionstatbestand entfallen muß und allein von völkerrechtsgeschichtlicher Bedeutung ist. Die angedeutete Befürchtung (S. 126, Fn. 507), der Jugoslawienkonflikt, insbesondere der Kampf um Bosnien-Herzegowina, könne durch Hinnahme gewaltsamer Gebietsveränderungen den erreichten Konsens zum Annexionsverbot gefährden, hat sich durch den nach Drucklegung geschlossenen Vertrag von Dayton beruhigenderweise nicht bewahrheitet. Ebenfalls großer Raum nimmt die praktisch oft komplizierte Abgrenzung der Inkorporation von der Fusion ein (ab S. 132). Zu Recht wird dem theoretisch eindeutigen Unterscheidungsmerkmal, daß die Fusion einen neuen Staat bildet, während die Inkorporation das Fortbestehen eines der beteiligten Staaten voraussetzt, die Frage entgegengehalten, wie das Abgrenzungsmerkmal der "Staatsidentität" (S. 132) praktisch mit Leben zu füllen ist. Eine Antwort hierauf soll die Berücksichtigung von Kriterien liefern, die "sich außerhalb der starren Rechtsfiguren des Völkerrechts bewegen" (S. 138) und am konkreten Einzelfall ermittelt werden müssen. Hier finden sich folgerichtig auch Ausführungen zu den Veränderungen im früheren Jugoslawien und der UdSSR; die Diskussion um deren Einstufung als Sezession oder Dismembration stellt das Gegenstück zur Abgrenzung von Inkorporation zu Fusion dar. Besonderes Gewicht als Indiz dafür, ob eine Staatsidentität vorliegt, kommt dem Willen der beteiligten Staaten zu, wobei nicht übersehen wird, daß im Falle des früheren Jugoslawiens der Wille "Restjugoslawiens" gerade nicht berücksichtigt wurde. Hier kollidiert der Wille der unmittelbar beteiligten Staaten mit der Bewertung durch die Staatengemeinschaft, der ebenfalls Indizcharakter zugesprochen wird. Dogmatisch feinsinnig schließt sich der Autor hier der (herrschenden) deklaratorischen Anerkennungslehre an, um zugleich zu betonen, daß in einer Völkerrechtswirklichkeit, die ihre Regeln zu einem nicht unerheblichen Teil aus dem Verhalten und der opinio iuris der handelnden Staaten zieht, auch deklaratorische Äußerungen von Anerkennung nicht völlig folgenlos sein können. Nachfolgend verwirrt die Trennung in "ausdrückliche" (S. 167) und "implizite" (S. 172) Bewertung von Staatenver- 
änderungen durch die Staatengemeinschaft etwas, insbesondere da sich auch die implizite Bewertung auf ausdrückliche Äußerungen der Staaten stützt. Die etwas künstlich wirkende Trennung ist wohl auch der tatsächlichen Uneinheitlichkeit des Staatenverhaltens in der Reaktion auf den Zerfall Jugoslawiens und der UdSSR geschuldet. Gesamtergebnis der Prüfung ist, daß für die Abgrenzung zwischen Inkorporation und Fusion "keine für jeden Fall passenden völkerrechtlichen Regeln, sondern nur das im Einzelfall ausfüllungsbedürftige Kriterium der Staatsidentität zur Verfügung steht" (S. 177). Daß die Einordnung der Indizien für oder gegen Staatsidentität - wie der Verfasser offen zugibt - je nach Betrachter wechseln kann, anscheinend ohne daß dies für die internationale Praxis zu unterschiedlichen Rechtsfolgen führt, läßt den Leser kurz am praktischen Wert des Versuchs einer sauberen dogmatischen Trennung zweifeln, ohne daß damit einem abstrakten dogmatischen Grundgerüst als grundsätzlichem Leitfaden die Berechtigung abgesprochen werden soll.

Den Abschluß des ersten Teils bilden eine kürzere Auseinandersetzung und Abgrenzung zur Zession, die die Souveränität über Gebietsteile, nicht über vollständige Staaten überträgt, die (aus der gefundenen Inkorporationsdefinition nur folgerichtige) Ablehnung einer separaten Fallgruppe des "Eintritts in einen Bundesstaat" und die Abgrenzung zu Gebietsveränderungen innerhalb eines Bundesstaates. Hervorzuheben sind die nachfolgend diskutierten Fallgruppen der "inkorporationsähnlichen Eingliederungen" (ab S. 183), die bei sonst gleichbleibender Definition des Tatbestandes das Inkorporationsobjekt anders fassen: Es handelt sich hier nicht um einen Staat, sondern einen autonom am Völkerrechtsverkehr teilnehmenden Gebietsteil eines anderen Staates, ein von einem anderen oder dem eigenen Staat abhängiges Gebiet oder eine "andere nicht-souveräne Gebietseinheit". Angesichts der Vielzahl von "Einheiten", die im Laufe der Völkerrechtsentwicklung am Völkerrechtsverkehr teilnehmen, ohne die Staatsdefinition zu erfüllen, sind gerade diese Fallgruppen für die Einordnung der Staatenpraxis unter den Inkorporationsbegriff von nicht zu unterschätzender Bedeutung.

Im zweiten Teil leistet der Autor durch die Sichtung der möglichen Anwendungsfälle der Inkorporation im "modernen" staatenzentrierten Völkerrecht (seit dem Westfälischen Frieden 1648) eine enorme Fleißarbeit und schafft eine solide Basis für den Interessierten, anhand der gesicherten Staatenpraxis aus dem nunmehr fest abgegrenzten Tatbestand der Inkorporation Folgerungen für die Existenz völkergewohnheitsrechtlicher Rechtsfolgenregelungen zu ziehen. Der Leser bleibt, angeregt von der überzeugenden dogmatischen Aufarbeitung des Tatbestandes und in Ansehung der sich auftürmenden Staatenpraxis, in der hoffnungsvollen Erwartung zurück, es möge sich bald jemand diese Vorarbeiten zunutze machen und eine ähnlich qualitätvolle Darstellung zu den Rechtsfolgen der Inkorporation abliefern.

Christiane Simmler 